\title{
Publisher Correction: Rational discovery of molecular glue degraders via scalable chemical profiling
}

Cristina Mayor-Ruiz, Sophie Bauer, Matthias Brand, Zuzanna Kozicka, Marton Siklos, Hana Imrichova (D), Ines H. Kaltheuner, Elisa Hahn (D), Kristina Seiler, Anna Koren (D), Georg Petzold, Michaela Fellner, Christoph Bock (1D, André C. Müller, Johannes Zuber (D), Matthias Geyer, Nicolas H. Thomä, Stefan Kubicek and Georg E. Winter (D)

Correction to: Nature Chemical Biology https://doi.org/10.1038/s41589-020-0594-x, published online 3 August 2020.

In the version of this Article originally published, cell line SKW3 was incorrectly referred to as SKN3 in Extended Data Fig. 10a-c, the Reporting Summary and the Methods section of the text. In the Methods, one instance was in the 'Cell lines and generation of mutants' section and the other was in the 'Cyclin $\mathrm{K}$ degradation-sensitivity correlations and synergy experiments' section. Additionally, panel letters a-i were missing in Fig. 3. The errors have been corrected.

Published online: 15 January 2021

https://doi.org/10.1038/s41589-021-00735-4

๑ The Author(s), under exclusive licence to Springer Nature America, Inc. 2021

\section{Addendum: MCC950 closes the active conformation of NLRP3 to an inactive state}

Ana Tapia-Abellán, Diego Angosto-Bazarra, Helios Martínez-Banaclocha, Carlos de Torre-Minguela, Jose P. Cerón-Carrasco, Horacio Pérez-Sánchez, Juan I. Arostegui and Pablo Pelegrin (D)

Addendum to: Nature Chemical Biology https://doi.org/10.1038/s41589-019-0278-6, published online 13 May 2019

In the version of this Brief Communication originally published, the compound used in Fig. 2a and Supplementary Fig. 4 was misrepresented as MCC950, while it was in fact a closely related derivative. The presented data were updated from those newly collected using the intended MCC950 compound. Furthermore, the sentence "After 100 ns of MD simulations, cluster 2 residues within Walker B presented a more favorable binding energy to MCC950 (Supplementary Fig. 4d), with F304 establishing stacking interactions with F257 and the aromatic ring of MCC950 (Supplementary Fig. 4b,e)" in the main text would have read "After 100 ns of MD simulations, cluster 2 residues within Walker B presented a more favorable binding energy to MCC950 (Supplementary Fig. 4d), with F257 establishing stacking and hydrophobic interactions with the aromatic ring of MCC950 (Supplementary Fig. 4b,e)," given the data on the proper MCC950 compound. A revised Fig. 2a and Supplementary Information PDF are included in the Addendum.

Published online: 25 January 2021

https://doi.org/10.1038/s41589-021-00741-6

$\odot$ The Author(s), under exclusive licence to Springer Nature America, Inc. 2021 